\title{
Maternal iron supplementation in pregnancy and asthma in the offspring: follow-up of a randomised trial in Finland
}

Seif O Shaheen ${ }^{1}$, Mika Gissler ${ }^{2,3}$, Graham Devereux ${ }^{4}$, Maijaliisa Erkkola ${ }^{5}$, Tarja I Kinnunen ${ }^{6}$, Harry Mcardle ${ }^{7}$, Aziz Sheikh ${ }^{8}$, Elina Hemminki², Bright I Nwaru ${ }^{6,8-10}$.

${ }^{1}$ Institute of Population Health Sciences, Barts and The London School of Medicine and

Dentistry, London, Queen Mary University of London, UK.

${ }^{2}$ Finnish Institute for Health and Welfare, Helsinki, Finland.

${ }^{3}$ Department of Neurobiology, Care Sciences and Society, Karolinska Institute, Stockholm, Sweden.

${ }^{4}$ Liverpool School of Tropical Medicine, Liverpool, UK.

${ }^{5}$ Department of Food and Nutrition, University of Helsinki, Helsinki, Finland.

${ }^{6}$ Unit of Health Sciences, Faculty of Social Sciences, Tampere University, Tampere, Finland

${ }^{7}$ Rowett Institute of Nutrition and Health, University of Aberdeen, Aberdeen, UK.

${ }^{8}$ Asthma UK Centre for Applied Research, Usher Institute, University of Edinburgh, Edinburgh, UK.

${ }^{9}$ Krefting Research Centre, Institute of Medicine, University of Gothenburg, Gothenburg, Sweden.

${ }^{10}$ Wallenberg Centre for Molecular and Translational Medicine, University of Gothenburg, Sweden

Correspondence and reprint requests to: Prof Seif Shaheen, Institute of Population Health Sciences, Barts and The London School of Medicine and Dentistry, 58 Turner Street, London E1 2AB. Email: s.shaheen@qmul.ac.uk

Tel: +44 (0)20 7882 2480; Fax: +44 (0)20 78822552

Word count: 1234 
To the Editor:

Evidence for the hypothesis that prenatal nutrition may influence the risk of childhood respiratory and atopic disorders has strengthened in recent years. For example, randomised controlled trials (RCTs) have suggested that maternal supplementation with vitamin D or fish oil in pregnancy could prevent early childhood wheezing and asthma in the offspring ${ }^{1 ; 2}$. Another nutrient of interest is iron. Animal experiments have implicated iron in fetal airway development $^{3}$. In two UK population-based birth cohorts, using different approaches, we have found evidence suggesting that lower maternal iron levels in pregnancy may increase the risk of wheezing, atopy and lower lung function in the offspring ${ }^{4-7}$. However, the possibility that unmeasured or residual confounding might explain these observational findings cannot be ruled out.

To date, no RCT has been conducted to test whether prenatal iron is causally related to childhood respiratory and atopic outcomes, and whether increasing maternal iron levels in pregnancy could improve the respiratory health of offspring. Such a trial would be timeconsuming and expensive. A faster, more efficient, approach is to opportunistically use a previous RCT of iron supplementation in pregnancy, originally undertaken with other outcomes in mind, and to measure respiratory/allergic outcomes in the offspring ${ }^{8}$. We followed up the offspring of mothers who took part in a population-based, multi-centre, RCT of iron supplementation in pregnancy in Finland, using health register linkage to investigate whether prenatal iron influenced their risk of asthma. Full details of the trial have been reported previously ${ }^{9-13}$. Pregnant women were recruited during 1985-1986, and 2,944 women were initially randomised, in each of 27 centres, to routine versus selective iron supplementation. After subsequent exclusions (e.g., twins) and miscarriages, 1,336 and 1,358 women in each arm, respectively, were included in the original analyses of maternal and perinatal outcomes $^{9 ; 13}$. The routine iron (RI) group received $100 \mathrm{mg}$ elemental iron/ day 
throughout pregnancy, regardless of haemoglobin concentration, which was usual care in Finland at the time. The selective iron (SI) group received $50 \mathrm{mg}$ elemental iron twice a day, but only if women were diagnosed as anaemic; this continued for two months or until their haemoglobin increased to $110 \mathrm{~g} / \mathrm{L}^{10 ; 11 ; 13}$. Compliance as reported by the mothers was good ${ }^{10}$. Haematocrit was measured at 12, 20, 28, and 36 weeks gestation, and information was collected on maternal factors, gestational age and weight at birth, and sex of child. Using unique personal identification numbers assigned to all Finnish residents, the RCT data were linked to national registers to ascertain the asthma status of the offspring of participating mothers. An asthma diagnosis was defined if an individual was found in the Register for the right to special reimbursement of medication and in the Register of reimbursed medication, maintained by the Social Insurance Institution (SII), and also had purchased asthma medication according to the Drug Prescription Register (Anatomical Therapeutic Chemical R03). To be eligible for the special reimbursement for purchase of asthma medication, a physician had to sign a certificate confirming an asthma diagnosis and the need for long-term treatment. Given that the registration for reimbursed medication started in 1995, an asthma diagnosis was only ascertained between 10-32 years of age. Additionally, using the inpatient admissions register, maintained since 1967 by the Finnish Institute for Health and Welfare, Helsinki, we defined first asthma admission (primary or secondary reason for admission) using the International Classification of Diseases (ICD) codes: ICD-8 (4930A, 4930B, 4931B, 4939X) up to 1995; ICD-9 (J45-J46) between 1996 and 2017. Researchers abstracting these data were blind to treatment allocation. We analysed associations between trial arm and asthma diagnosis based on the SII reimbursement data (primary outcome) and asthma admissions (secondary outcome) using random-effects models (multilevel mixed-effects generalised linear model for the former, and multilevel mixed-effects survival model for the latter outcome). This allowed effects to vary between trial centres, given that randomisation 
was stratified by centre ${ }^{14}$. Risk ratios and hazard ratios, respectively, were adjusted for prognostic factors known to be associated with childhood asthma risk, namely, maternal factors (age, pre-pregnancy body mass index, smoking and asthma) and sex of child, in order to increase statistical power ${ }^{15}$. Analyses were by intention-to-treat, using Stata version 15 (StataCorp, College Station, TX, USA).

Of the 2,694 women included in the original trial analysis, 2,666 (99\%) were included in our follow-up analysis (1,320 in the RI arm and 1,346 in the SI arm). The Figure shows mean haematocrit in pregnancy by gestational age and trial arm. In the last two months of pregnancy, haematocrit increased substantially more in the RI group than in the SI group (difference at 36 weeks gestation $\mathrm{P}<0.001$ ). There was no imbalance between trial arms in most background characteristics, but mothers in the RI arm were more likely to be underweight.

During the follow-up period, there were 74 individuals with doctor-diagnosed asthma based on the SII data, and 138 first-time hospital admissions for asthma. Compared to offspring in the SI arm, those in the RI arm had a significant reduction in risk of asthma diagnosis (adjusted Risk Ratio 0.58 (95\% CI: 0.35 to 0.96), $\mathrm{P}=0.033$ ) and a non-significant reduction in risk of asthma admissions (adjusted Hazard Ratio 0.86 (0.61 to 1.22), $\mathrm{P}=0.41$ ). As there was a minor reduction in the prevalence of premature birth in the RI group compared to the SI group, we repeated the analyses with further adjustment for gestational age at birth, but this did not alter the risk estimates.

This study has a number of strengths. First, the association between routine iron supplementation in pregnancy and reduced risk of asthma diagnosis in the offspring is unlikely to be confounded, given that the intervention was randomised. Second, the increase in haematocrit in mothers receiving routine supplementation, which was not seen in the selective supplementation group, confirms compliance with supplementation, suggesting that 
prenatal iron status is likely to have been significantly higher in the former group during the latter stages of pregnancy. With respect to asthma ascertainment, individuals identified in the reimbursement register are likely to represent the most severe asthma cases, given the strict criteria for reimbursement eligibility, so mild cases may not have been identified. Also, as the reimbursed medication register did not start until 1995, we could not ascertain an asthma diagnosis until the offspring were 10 years old, hence some cases under that age may not have been identified unless their condition persisted beyond that age. This limited our ability to assess the effect of iron supplementation on the incidence of early onset of disease, when most wheeze and asthma begins. In our experience, those admitted with 'asthma' to hospital are likely to include some individuals with acute infective episodes e.g. acute bronchitis, in whom a firm diagnosis of asthma is not confirmed subsequently. We therefore believe that ascertainment of an asthma diagnosis using the reimbursement register will have led to less misclassification of asthma, compared to asthma ascertained using the admissions register, and this may explain the stronger findings for the former asthma outcome. On the other hand, we acknowledge that the reimbursement register approach will have led to underascertainment of mild cases and asthma beginning before 10 years of age. Importantly though, any misclassification of asthma is likely to be random (non-differential) with respect to trial arm, and this would tend to lead to an underestimation of effect. We also acknowledge that a trial comparing routine prenatal iron supplementation versus placebo would have enabled a more powerful test of our hypothesis; if the effects on asthma risk are causal we would expect such a trial to produce a larger effect than we observed in our study comparing two supplementation regimes. Whilst maternal iron deficiency is associated with lower birth weight and prematurity ${ }^{16}$, which are both risk factors for asthma, there was little impact of routine versus selective supplementation on these perinatal outcomes in this trial. Hence, they did not appear to mediate the reduction in risk of asthma associated with routine iron 
supplementation. We speculate that improving prenatal iron status may have reduced the risk of more severe asthma by optimising airway growth.

In conclusion, these findings suggest that routine iron supplementation may reduce the risk of asthma in the offspring, and could therefore offer a potential primary prevention strategy. Additional robust, randomised evidence from a low- or middle-income setting, where iron deficiency anaemia in pregnancy continues to be a major public health problem, would be of interest.

\section{Acknowledgements}

This study was funded by the Tampere Tuberculosis Foundation. BN acknowledges the support of the Knut and Alice Wallenberg Foundation, the Wallenberg Centre for Molecular and Translational Medicine, and the VBG Group Herman Krefting Foundation on Asthma and Allergy. AS is supported by the Asthma UK Centre for Applied Research. Ethics approval was obtained from the National Institute for Health and Welfare.

Figure: Haematocrit in pregnancy by gestational age and trial arm. 


\section{References}

(1) Wolsk HM, Chawes BL, Litonjua AA, Hollis BW, Waage J, Stokholm J et al. Prenatal vitamin D supplementation reduces risk of asthma/recurrent wheeze in early childhood: A combined analysis of two randomized controlled trials. PLOS ONE 2017; 12: 0186657.

(2) Bisgaard H, Stokholm J, Chawes BL, Vissing NH, Bjarnadottir E, Schoos AM et al. Fish Oil-Derived Fatty Acids in Pregnancy and Wheeze and Asthma in Offspring. $N$ Engl $J$ Med 2016; 375:2530-2539.

(3) Groenman FA, Rutter M, Wang J, Caniggia I, Tibboel D, Post M. Effect of chemical stabilizers of hypoxia-inducible factors on early lung development. Am J Physiol - Lung Cell Molec Physiol 2007; 293:L557-L567.

(4) Shaheen SO, Newson RB, Henderson AJ, Emmett PM, Sherriff A, Cooke M et al. Umbilical cord trace elements and minerals and risk of early childhood wheezing and eczema. Eur Respir J 2004; 24:292-297.

(5) Shaheen SO, Macdonald-Wallis C, Lawlor DA, Henderson AJ. Haemoglobin concentrations in pregnancy and respiratory and allergic outcomes in childhood: Birth cohort study. Clin Exp Allergy 2017; 47:1615-1624.

(6) Nwaru BI, Hayes H, Gambling L, Craig LCA, Allan K, Prabhu N et al. An exploratory study of the associations between maternal iron status in pregnancy and childhood wheeze and atopy. Br J Nutr 2014; 112:2018-2027.

(7) Bedard A, Lewis SJ, Burgess S, Henderson AJ, Shaheen SO. Maternal iron status during pregnancy and respiratory and atopic outcomes in the offspring: a Mendelian randomisation study. BMJ Open Resp Res 2018; 5(1).

(8) Shaheen SO. Prenatal nutrition and asthma: hope or hype? Thorax 2008; 63:483-485. 
(9) Hemminki E, Rimpela U. Iron supplementation, maternal packed cell volume, and fetal growth. Arch Dis Child 1991; 66:422-425.

(10) Hemminki E, Uski A, Koponen P, Rimpela U. Iron supplementation during pregnancy - experiences of a randomized trial relying on health service personnel. Control Clin Trials 1989; 10:290-298.

(11) Hemminki E, Merilainen J. Long-term follow-up of mothers and their infants in a randomized trial on iron prophylaxis during pregnancy. Am J Obstet Gynecol 1995; 173:205209.

(12) Hemminki E, Rimpela U. A randomized comparison of routine versus selective iron supplementation during pregnancy. J Am Coll Nutr 1991; 10:3-10.

(13) Kinnunen TI, Luoto R, Helin A, Hemminki E. Supplemental iron intake and the risk of glucose intolerance in pregnancy: re-analysis of a randomised controlled trial in Finland. Matern Child Nutr 2016; 12:74-84.

(14) Kahan BC. Accounting for centre-effects in multicentre trials with a binary outcome when, why, and how? BMC Med Res Methodol 2014; 14:20.

(15) Kahan BC, Jairath V, Dore CJ, Morris TP. The risks and rewards of covariate adjustment in randomized trials: an assessment of 12 outcomes from 8 studies. Trials 2014; $15: 139$

(16) Haider BAO. Anaemia, prenatal iron use, and risk of adverse pregnancy outcomes: systematic review and meta-analysis. BMJ 2013; 346. 